\title{
FAULT DETECTION IN NONLINEAR SYSTEMS VIA LINEAR METHODS
}

\author{
ALEXEY ZHIRABOK ${ }^{a, b, *}$, ALEXEY SHUMSKY ${ }^{a, c}$, SERGEY SOLYANIK ${ }^{a}$, AlEXEY SUVOROV $^{a}$
}

\author{
${ }^{a}$ Department of Automation and Control \\ Far Eastern Federal University, Sukhanova, 8, Vladivostok, 690990, Russia \\ e-mail: zhirabok@mail.ru \\ ${ }^{b}$ Department of Robotic Systems \\ Institute of Marine Technology Problems, Sukhanova, 5, Vladivostok, 690990, Russia \\ ${ }^{c}$ Department of Automatic Control \\ Institute of Applied Mathematics, Radio, 5, Vladivostok, 690014, Russia \\ e-mail: a.e.shumsky@gmail.com
}

\begin{abstract}
The problem of robust linear and nonlinear diagnostic observer design is considered. A method is suggested to construct the observers that are disturbance decoupled or have minimal sensitivity to the disturbances. The method is based on a logic-dynamic approach which allows us to consider systems with non-differentiable nonlinearities in the state equations by methods of linear algebra.
\end{abstract}

Keywords: nonlinear dynamic systems, diagnostic observers, robustness, non-differentiable nonlinearities, logic-dynamic approach.

\section{Introduction and problem statement}

The problem of fault diagnosis has extensively been investigated for the past 30 years; see, e.g., the surveys by Alcorta-Garcia and Frank (1997), Gertler (1993), Frank (1990), Patton (1994), Samy (2011), and the books by Blanke et al. (2006), Ding (2013), Caccavale and Villiani (2002), Patton et al. (2000), Russell et al. (2001), or Simani et al. (2002). Various models of technical systems have been investigated: linear, polynomial, nonlinear, descriptor, and hybrid. Many practical examples have been considered; in particular, special books have been devoted to industrial systems (Boulkroune et al., 2013; Caccavale and Villiani, 2002; Ducard, 2015; Filaretov et al., 2003; Russell et al., 2001).

The main purpose of this paper is to consider the fault diagnosis problem for a wide class of technical systems, in particular, mechatronic systems. As a rule, these systems are nonlinear essentially with non-differentiable nonlinearities such as saturation, Coulomb friction, backlash, and hysteresis. In this case, one has to use nonlinear methods of diagnosis. However, most

\footnotetext{
*Corresponding author
}

of papers dealing with the fault diagnosis problem consider the nonlinear systems with smooth nonlinearities (Alcorta-Garcia and Frank, 1997; Persis and Isidori, 2001; Schreier et al., 1997). Therefore, they cannot be used in this case. There are several papers developing the algebraic approach to the fault diagnosis problem and intended for systems with non-differentiable nonlinearities (Shumsky and Zhirabok, 2006; Zhirabok et al., 2010). However, they require rather complex analytical transformations and, therefore, it is difficult to use them in practice.

This paper is intended to overcome these difficulties and develop an approach allowing one to solve the fault diagnosis problem for a wide class of industrial and robotic systems described by nonlinear dynamic models. The so-called logic-dynamic approach (LDA) is considered to obtain a simple fault diagnosis procedure in systems with non-differentiable nonlinearities. The LDA has been suggested by Zhirabok and Usoltsev (2002) and used for various applications by Filaretov and Zhirabok (2006) or Zhirabok and Shumsky (2010). The main idea of this approach consists in replacing the initial nonlinear system by a linear one, solving the problem 
under consideration for this linear system by well-known linear methods and finally taking into account nonlinear terms to correct the obtained solution.

In comparison with the paper by Persis and Isidori (2001), where affine systems are considered and differential geometry is used for solution of the problem, the present paper deals with systems containing non-differentiable nonlinearities and uses linear algebra methods underlying the LDA.

The problem solved in this paper is to develop the LDA for fault detection in systems described by dynamic models with non-differentiable nonlinearities.

\section{Basic models and relations}

Consider a nonlinear control system described by the equations

$$
\begin{aligned}
x(t+1) & =f(x(t), u(t), \gamma(t))+L \rho(t), \\
y(t) & =H x(t),
\end{aligned}
$$

where $x(t) \in X \subseteq \mathbb{R}^{n}, u(t) \in U \subseteq \mathbb{R}^{m}, y(t) \in Y \subseteq \mathbb{R}^{l}$ are vectors of state, control, and output, respectively; $\rho(t)$ is an unknown function of time describing the disturbances; $\gamma(t) \in \mathbb{R}^{p}$ is the vector of parameters: if there are no faults, $\gamma(t)=\gamma_{0}$, where $\gamma_{0}$ is the nominal value of the vector $\gamma$, if the $i$-th fault occurs, the $i$-th component $\gamma_{i}(t)$ becomes an unknown function of time; $f$ is a nonlinear vector function which should be differentiable with respect to $\gamma$ and may be non-smooth with respect to $x$ and $u$; the matrices $L$ and $H$ are assumed to be constant. Additionally, we assume that the system (1) is stable.

According to the LDA, solution of the problem under consideration includes four main steps (Zhirabok and Usoltsev, 2002; Zhirabok and Shumsky, 2013).

\begin{tabular}{l}
\hline Algorithm 1. Logic-dynamic approach. \\
Step 1. Transforming the initial system (1) into the form \\
where the linear and nonlinear terms are separated.
\end{tabular}

Step 2. Replacing the transformed nonlinear system by a linear system via removing the nonlinear terms.

Step 3. Solving the problem under consideration for the obtained linear system with some additional restriction.

Step 4. Transforming the obtained linear observer into the nonlinear one by adding nonlinear terms to the model constructed in Step 3.

To implement the first step of the LDA, use the following approximate representation of the function $f(x(t), u(t), \gamma(t))$ :

$$
f(x, u, \gamma)=f\left(x, u, \gamma_{0}\right)+\sum_{i=1}^{p} \frac{\partial f(x, u, \gamma)}{\partial \gamma_{i}}\left(\gamma_{i}-\gamma_{0 i}\right)
$$

Write

$$
\begin{aligned}
& D_{i}:=\frac{\partial f(x, u, \gamma)}{\partial \gamma_{i}}, \\
& d_{i}:=\gamma_{i}-\gamma_{0 i}, \quad i=1,2, \ldots, p, \\
& D:=\left(\begin{array}{llll}
D_{1} & D_{2} & \ldots & D_{p}
\end{array}\right), \\
& d:=\left(\begin{array}{llll}
d_{1} & d_{2} & \ldots & d_{p}
\end{array}\right)^{T}, \\
& f(x, u):=f\left(x, u, \gamma_{0}\right)
\end{aligned}
$$

and rewrite the first equation in (1) in the form

$$
x(t+1)=f(x(t), u(t))+D(x(t), u(t)) d(t)+L \rho(t) .
$$

Then transform the function $f(x(t), u(t))$ by separating the linear terms from nonlinear ones. Such a transformation results in the system of the form

$$
\begin{aligned}
x(t+1)= & F x(t)+G u(t)+D(x(t), u(t)) d(t) \\
& +L \rho(t)+C\left(\begin{array}{c}
\varphi_{1}\left(A_{1} x(t), u(t)\right) \\
\vdots \\
\varphi_{q}\left(A_{q} x(t), u(t)\right)
\end{array}\right), \\
y(t)= & H x(t),
\end{aligned}
$$

where $F$ and $G$ are matrices of appropriate dimensions, describing the linear part of the system; $C$ is a constant matrix, $\varphi_{1}, \ldots, \varphi_{q}$ are arbitrary nonlinearities, $A_{1}, \ldots$, $A_{q}$ are row matrices. It is assumed that the pair $(F, H)$ is observable and the function $\varphi_{i}$ is Lipschitz with constant $M_{i}$, i.e.

$$
\left\|\varphi_{i}\left(A_{i} x, u\right)-\varphi_{i}\left(A_{i} x^{\prime}, u\right)\right\| \leq M_{i}\left|A_{i}\left(x-x^{\prime}\right)\right|,
$$

$i=1, \ldots, q$ (Schreier et al., 1997). Generally, the function $\varphi_{j}$ may contain several terms in the form $A_{j} x(t)$.

Example 1. Consider the control system (for simplicity, we use the notation: $x^{+}=x(t+1), x=x(t), u=u(t)$, $y=y(t)$ :

$$
\begin{aligned}
& x_{1}^{+}=\ln \left|x_{1}\right|-2 x_{1}+u_{2}+x_{2}+x_{2} u_{1}^{2}, \\
& x_{2}^{+}=-x_{2}-\ln \left|x_{1}\right|-u_{1} .
\end{aligned}
$$

Clearly, the matrices and the function used in (2) are given by

$$
\begin{aligned}
& F=\left(\begin{array}{cc}
-2 & 1 \\
0 & -1
\end{array}\right), \quad G=\left(\begin{array}{cc}
0 & 1 \\
-1 & 0
\end{array}\right) \\
& C=\left(\begin{array}{cc}
1 & 1 \\
-1 & 0
\end{array}\right) \\
& \varphi_{1}\left(A_{1} x, u\right)=\ln \left|A_{1} x\right| \text {, } \\
& \varphi_{2}\left(A_{2} x, u\right)=\left(A_{2} x\right) u_{1}^{2} \text {, } \\
& A_{1}=\left(\begin{array}{ll}
1 & 0
\end{array}\right) \text {, } \\
& A_{2}=\left(\begin{array}{ll}
0 & 1
\end{array}\right) \text {. }
\end{aligned}
$$


In some cases, removing the nonlinear terms in Step 2 of the LDA may yield dynamics of the linear part of system (2) such that no solution in Step 3 exists. To overcome this difficulty, we need to make some corrections in the model (2).

Example 2. (Continuation of Example 1) Notice that according to the structure of the matrix $F$ above, the linear part does not contain the variable $x_{1}(t)$ on the right-hand side of the equation for $x_{2}^{+}$while the initial system does. To correct this situation, insert the formal term $x_{1}(t)-x_{1}(t)$ into the right-hand side of the second equation and include the variable $x_{1}(t)$ in the linear part, $-x_{1}(t)$ in the nonlinear term. As a result, the matrix $F$ and the nonlinear term become

$$
F=\left(\begin{array}{cc}
-3 & 1 \\
1 & -1
\end{array}\right)
$$

and $\varphi_{1}\left(A_{1} x(t), u(t)\right)=\ln \left|A_{1} x(t)\right|+x_{1}(t)$, respectively.

Generally, if some equation in the model (2) contains some variable $x_{j}$ on its right-hand side only as an argument of some nonlinear function, the formal term $x_{j}-x_{j}$ should be inserted into this equation. Then the variable $x_{j}$ supplements the linear part and $-x_{j}$ the nonlinear term.

The model of the diagnostic observer is found in the form similar to the model (2) that is required for the LDA application:

$$
\begin{aligned}
x_{*}(t+1)= & F_{*} x_{*}(t)+G_{*} u(t)+J y(t) \\
& +C_{*}\left(\begin{array}{c}
\varphi_{1}\left(A_{* 1} z(t), u(t)\right) \\
\vdots \\
\varphi_{q}\left(A_{* q} z(t), u(t)\right)
\end{array}\right)+K r(t), \\
y_{*}(t)= & H_{*} x_{*}(t),
\end{aligned}
$$

where

$$
z(t)=\left(\begin{array}{c}
x_{*}(t) \\
y(t)
\end{array}\right)
$$

$K$ is the gain matrix, $x_{*}(t) \in \mathbb{R}^{k}$ is the state vector of the observer, the scalar residual $r(t)$ is generated as

$$
r(t)=R y(t)-y_{*}(t)
$$

for some row matrix $R$. The problems of the matrix $K$ choice are studied in (Schreier et al., 1997), and are not considered in this paper.

It is assumed that when the faults and disturbances are absent, the states $x_{*}(t)$ of the observer are linear combinations of the system states $x(t)$ according to

$$
x_{*}(t)=\Phi x(t)
$$

after the response to an unlikely condition has died out (Frank, 1990). A sufficient condition for the existence of such an "ideal" relation is the stability of the observer provided by the appropriate choice of the gain matrix $K$.

Notice that in the works of Persis and Isidori (2001) or Shumsky and Zhirabok (2006) the states $x_{*}(t)$ are nonlinear combinations of $x(t)$, generally. Our assumption limits the class of possible problem solutions, but allows us to use only the methods of linear algebra.

The matrix $\Phi$ satisfies the following well-known conditions (Frank, 1990; Zhirabok and Usoltsev, 2002):

$$
R H=H_{*} \Phi, \quad \Phi F=F_{*} \Phi+J H, \quad G_{*}=\Phi G
$$

associated with the linear parts of system (2) and the observer (3). The additional conditions

$$
C_{*}=\Phi C, \quad A=A_{*}\left(\begin{array}{c}
\Phi \\
H
\end{array}\right)
$$

correspond to the nonlinear terms (Zhirabok and Shumsky, 2013). Here

$$
A=\left(\begin{array}{c}
A_{1} \\
\vdots \\
A_{q}
\end{array}\right), \quad A_{*}=\left(\begin{array}{c}
A_{* 1} \\
\vdots \\
A_{* q}
\end{array}\right) .
$$

The last relation in (5) is equivalent to the condition

$$
\operatorname{rank}\left(\begin{array}{c}
\Phi \\
H
\end{array}\right)=\operatorname{rank}\left(\begin{array}{c}
\Phi \\
H \\
A
\end{array}\right) .
$$

Indeed, if (6) holds, then every row of the matrix $A$ linearly depends on the rows of the matrices $\Phi$ and $H$, that is a matrix $A_{*}$ exists such that the last relation in (5) holds. The implication $(5) \Rightarrow(6)$ is evident.

Besides, the matrix $\Phi$ has to satisfy the conditions

$$
\Phi L=0, \quad \Phi D_{i}(x(t), u(t)) \neq 0, \quad i=1, \ldots, p .
$$

The first is a full decoupling condition. This means that the residual $r(t)$ is disturbance decoupled; the second relates to the sensitivity to the faults. We admit that the second condition may be violated on a set of measure zero. Let $X=\mathbb{R}^{4}$ and $\Phi D(x(t), u(t))=\operatorname{abs}\left(x_{1}(t)+2 x_{3}(t)-\right.$ $\left.x_{4}(t)\right)$, then $\Phi D(x(t), u(t)) \neq 0$ for all $(x(t), u(t)) \in$ $X \times U$ except on the set $S=\{(x(t), u(t)) \in X \times$ $\left.U \mid x_{1}(t)+2 x_{3}(t)-x_{4}(t)=0, u(t) \in U\right\}$; the measure of the set $S$ is equal to zero.

Consider the linear part of system (2) and assume that the pair $\left(F_{*}, H_{*}\right)$ is observable. It is known that in this case the matrices $F_{*}$ and $H_{*}$ can be presented in the canonical form

$$
\begin{aligned}
F_{*} & =\left(\begin{array}{ccccc}
0 & 1 & 0 & \ldots & 0 \\
0 & 0 & 1 & \ldots & 0 \\
\ldots & \ldots & \ldots & \ldots & \ldots \\
0 & 0 & 0 & \ldots & 0
\end{array}\right), \\
H_{*} & =\left(\begin{array}{ccccc}
1 & 0 & 0 & \ldots & 0
\end{array}\right) .
\end{aligned}
$$


Here first the two equations in (4) can be expressed in the form of $k+1$ equations:

$$
\begin{aligned}
R H & =\Phi_{1}, \\
\Phi_{i} F & =\Phi_{i+1}+J_{i} H, \quad i=1, \ldots, k-1, \\
\Phi_{k} F & =J_{k} H,
\end{aligned}
$$

where $\Phi_{i}$ and $J_{i}$ are the $i$-th rows of the matrices $\Phi$ and $J$, respectively, $i=1, \ldots, k, k$ is the observer dimension. It was shown by Zhirabok and Usoltsev (2002) that (7) can be reduced to the single equation

$$
R H F^{k}=J_{1} H F^{k-1}+J_{2} H F^{k-2}+\cdots+J_{k} H .
$$

Solutions of this equation are the minimal dimension $k$ and the matrices $R$ and $J$.

Rewrite (8) in the form

$$
\left(\begin{array}{lllll}
R & -J_{1} & -J_{2} & \ldots & -J_{k}
\end{array}\right) V^{(k)}=0,
$$

where

$$
V^{(k)}=\left(\begin{array}{c}
H F^{k} \\
H F^{k-1} \\
\vdots \\
H
\end{array}\right) .
$$

This equation has nontrivial solutions if

$$
\operatorname{rank}\left(V^{(k)}\right)<l(k+1) .
$$

There are $M=l(k+1)-\operatorname{rank}\left(V^{(k)}\right)$ variants of solutions of (9) where the minimal value of the dimension $k$ can be found from the condition (10).

A shortcoming of the method considered is that it does not take into account the condition $\Phi L=0$. Therefore, after solving (9), we have to check this condition. If it does not hold, we have to find another solution to the former or to increase the dimension $k$, i.e., we must consider several variants.

To overcome this shortcoming, we develop a new method for solving the fault detection problem allowing to reduce the number of variants owing to taking account of the condition $\Phi L=0$.

\section{Observer existence conditions}

It is well known (Frank, 1990; Zhirabok and Usoltsev, 2002) that (9) is solvable for an arbitrary matrix $\mathbb{R}$, i.e., if this matrix is known, one can find the matrix $J$ in addition to the matrices $F_{*}$ and $H_{*}$ presented in the canonical form. The condition $\Phi L=0$ imposes a restriction on the possibility to design such an observer. Therefore, it is reasonable to check whether the observer with this property exists.

To this end, introduce the matrix $L^{0}$ of maximal row rank such that $L^{0} L=0$. Then $\Phi=N L^{0}$ for some matrix $N$.
Replace the matrix $\Phi$ by $\Phi=N L^{0}$ in the equation $R H=H_{*} \Phi$ and transform it by separating known matrices from unknown ones as follows:

$$
\left(\begin{array}{ll}
R & -H_{*} N
\end{array}\right)\left(\begin{array}{c}
H \\
L^{0}
\end{array}\right)=0 .
$$

Equation (11) has a nontrivial solution with $\mathbb{R} \neq 0$ when the rows of the matrices $H$ and $L^{0}$ are linearly dependent. This is equivalent to the following condition:

$$
\operatorname{rank}\left(\begin{array}{c}
L^{0} \\
H
\end{array}\right)<\operatorname{rank}(H)+\operatorname{rank}\left(L^{0}\right) .
$$

As for the equation $\Phi F=F_{*} \Phi+J H$, replacing the matrix $\Phi$ by $\Phi=N L^{0}$ and transforming the result, we obtain

$$
\left(\begin{array}{lll}
N & -F_{*} N & -J
\end{array}\right)\left(\begin{array}{c}
L^{0} F \\
L^{0} \\
H
\end{array}\right)=0 .
$$

This equation has a nontrivial solution with $N \neq 0$ when rows of the matrices $L^{0} F$ and $L^{0}$ are linearly dependent. Clearly, (13) is true if

$$
\operatorname{rank}\left(\begin{array}{c}
L^{0} F \\
L^{0}
\end{array}\right)<\operatorname{rank}\left(L^{0} F\right)+\operatorname{rank}\left(L^{0}\right) .
$$

Considering by analogy the equation

$$
A=A_{*}\left(\begin{array}{c}
\Phi \\
H
\end{array}\right)
$$

we get the necessary condition

$$
\operatorname{rank}\left(\begin{array}{c}
L^{0} \\
H
\end{array}\right)=\operatorname{rank}\left(\begin{array}{c}
L^{0} \\
H \\
A
\end{array}\right) .
$$

Note that the inequalities (12), (14), and (15) are necessary solvability conditions for the problem of designing a disturbance decoupled observer.

The condition (14) is not sufficient because if the matrix ( $\left.\begin{array}{ccc}A^{\prime} & B^{\prime} & C^{\prime}\end{array}\right)$ is a solution of (13), i.e.,

$$
\left(\begin{array}{lll}
A^{\prime} & B^{\prime} & C^{\prime}
\end{array}\right)\left(\begin{array}{c}
L^{0} F \\
L^{0} \\
H
\end{array}\right)=0,
$$

then rows of the matrix $B^{\prime}$ must be linearly dependent on the rows of the matrix $A^{\prime}$ according to (13). The condition (15) is not sufficient since the rows of the matrix $A$ may be linearly independent of the matrices $\Phi=N L^{0}$ and the rows of $H$, even if the rows of the matrix $A$ are linearly dependent on the matrices $L^{0}$ and the rows of $H$.

If one of these conditions is not valid, the disturbance decoupled observer cannot be built, and one has to use robust methods. If, however, all conditions are valid, this 
does not guarantee the existence of such an observer but means only a potential for the observer design.

In addition to the condition $\Phi L=0$, the condition $\Phi D_{i}(x(t), u(t)) \neq 0$ for all $i=1, \ldots, p$ has to be checked as well. To detect faults, this condition should be satisfied for all $(x(t), u(t)) \in X \times U$ except on a set of measure zero. Notice that this condition is not very rigid unlike $\Phi L=0$.

\section{Full decoupling}

In this section, we solve the problem of the diagnostic observer design when the conditions $\Phi L=0$ and $\Phi D_{i}(x(t), u(t)) \neq 0$ hold for all $i=1, \ldots, p$ i.e., the observer is disturbance decoupled and sensitive to faults.

4.1. Linear systems. In this section, we assume the conditions (12), (14), and (15) are valid.

Based on (7), obtain the equations for the first three rows of the matrix $\Phi$ :

$$
\begin{aligned}
\Phi_{1} & =R H, \\
\Phi_{2} & =\Phi_{1} F-J_{1} H=R H F-J_{1} H, \\
\Phi_{3} & =\Phi_{2} F-J_{2} H=\left(R H F-J_{1} H\right) F-J_{2} H \\
& =R H F^{2}-J_{1} H F-J_{2} H .
\end{aligned}
$$

The relations for the rest of the rows can be obtained by analogy.

It is known (Alcorta-Garcia and Frank, 1997) that the contribution of the disturbance to the residual can be estimated by the Frobenius norm of the matrix $\Phi L$ as follows:

$$
\|\Phi L\|_{F}=\left(\sum_{i=1}^{k} \sum_{j=1}^{b}(\Phi L)_{i j}^{2}\right)^{1 / 2}
$$

where $b$ is the number of columns of the matrix $L$. From (17) it follows that $\|\Phi L\|_{F}^{2}=\sum_{i=1}^{k}\left\|\Phi_{i} L\right\|_{F}^{2}=\left\|\Phi_{*}\right\|_{F}^{2}$, where

$$
\Phi_{*}=\left(\begin{array}{llll}
\Phi_{1} L & \Phi_{2} L & \ldots & \Phi_{k} L
\end{array}\right) .
$$

The last relation allows us to replace the product $\Phi L$ in (17) by $\Phi_{*}$. Replace the elements in $\Phi_{*}$ according to (16):

$$
\begin{aligned}
\Phi_{*}=\left(\begin{array}{ll}
R H L & R H F L-J_{1} H L \\
& R H F^{2} L-J_{1} H F L-J_{2} H L \quad \ldots
\end{array}\right)
\end{aligned}
$$

It is easy to check that the last matrix can be rewritten in the form

$$
\Phi_{*}=\left(\begin{array}{llll}
R & -J_{1} & \ldots & -J_{k}
\end{array}\right) B_{1}^{(k)},
$$

where

$$
B_{1}^{(k)}=\left(\begin{array}{ccccc}
H L & H F L & H F^{2} L & \ldots & H F^{k-1} L \\
0 & H L & H F L & \ldots & H F^{k-2} L \\
0 & 0 & H L & \ldots & H F^{k-3} L \\
\ldots \ldots & \ldots \ldots \ldots \ldots \ldots \ldots \ldots \ldots \ldots \ldots \\
0 & 0 & 0 & \ldots & 0
\end{array}\right) .
$$

The condition $\Phi L=0$ is now of the form $\left(\begin{array}{llll}R & -J_{1} & \ldots & -J_{k}\end{array}\right) B_{1}^{(k)}=0$. Apart from that, the matrix $\left(\begin{array}{llll}R & -J_{1} & \ldots & -J_{k}\end{array}\right)$ satisfies the condition (9) therefore, we obtain

$$
\left(\begin{array}{llll}
R & -J_{1} & \ldots & -J_{k}
\end{array}\right)\left(\begin{array}{ll}
V^{(k)} & B_{1}^{(k)}
\end{array}\right)=0 .
$$

The minimal value of the dimension $k$ can be found through the condition similar to (10):

$$
\operatorname{rank}\left(V^{(k)} B_{1}^{(k)}\right)<l(k+1)
$$

Whenever (20) is satisfied, a row vector $\left(\begin{array}{llll}R & -J_{1} & \ldots & -J_{k}\end{array}\right)$ exists such that (19) has a solution. Then the rows of the matrix $\Phi$ are obtained from (16) and the matrix $G_{*}=\Phi G$ is determined. As a result, the disturbance decoupled linear observer has been built. If $\Phi D_{i}(x(t), u(t))=0$ for some $i$ and for all $(x(t), u(t)) \in X \times U$, another solution of (19) should be found.

Thus, the suggested approach allows us to obtain a solution in the linear case without detailed analysis of several variants, one needs to find the minimal dimension $k$ only. A more sophisticated analysis is required in the nonlinear case.

4.2. Nonlinear systems. Notice that if the rows of the matrix $A$ in (2) linearly depend on the matrix $H$, the argument of the nonlinear function in (3) depends on the output vector $y$ only and the problem of the nonlinear observer design reduces to the linear case. To analyze the general case, consider the set of all linearly independent solutions of (19) for some $k$ presented in the form

$$
W=\left(\begin{array}{cccc}
R^{(1)} & -J_{1}^{(1)} & \ldots & -J_{k}^{(1)} \\
\ldots \ldots & \ldots & \ldots & \ldots \\
R^{(N)} & -J_{1}^{(N)} & \ldots & -J_{k}^{(N)}
\end{array}\right)
$$

$N$ is the number of such solutions.

Theorem 1. Let the matrices $\Phi^{(1)}, \ldots, \Phi^{(N)}$ obtained based on (21) describe the set of linearly independent disturbance decoupled solutions for the linear system. Then any arbitrary linear combination of solutions from (21) yields the matrix $\Phi$ which is the appropriate linear combination of the matrices $\Phi^{(1)}, \ldots, \Phi^{(N)}$ and describes some disturbance decoupled solution as well. 
Proof. Let

$$
\begin{aligned}
&\left(\begin{array}{llll}
R & -J_{1} & \ldots & -J_{k}
\end{array}\right) \\
&=v_{1}\left(\begin{array}{lllll}
R^{(1)} & -J_{1}^{(1)} & \ldots & -J_{k}^{(1)}
\end{array}\right) \\
&+v_{2}\left(\begin{array}{llll}
R^{(2)} & -J_{1}^{(2)} & \ldots & -J_{k}^{(2)}
\end{array}\right)
\end{aligned}
$$

be some linear combination of two solutions, where $v_{1}$ and $v_{2}$ are weight coefficients then $R=v_{1} R^{(1)}+v_{2} R^{(2)}$, $J_{1}=v_{1} J_{1}^{(1)}+v_{2} J_{1}^{(2)}, \ldots, J_{k}=v_{1} J_{k}^{(1)}+v_{2} J_{k}^{(2)}$. Since (19) is a homogeneous equation, the row matrix $\left(\begin{array}{llll}R & -J_{1} & \ldots & -J_{k}\end{array}\right)$ is also the solution of (19). Then

$$
\begin{aligned}
\Phi_{1}= & R H=v_{1} R^{(1)} H+v_{2} R^{(2)} H \\
= & v_{1} \Phi_{1}^{(1)}+v_{2} \Phi_{1}^{(2)} \\
\Phi_{2}= & \Phi_{1} F-J_{1} H \\
= & \left(v_{1} \Phi_{1}^{(1)}+v_{2} \Phi_{1}^{(2)}\right) F-\left(v_{1} J_{1}^{(1)}+v_{2} J_{1}^{(2)}\right) \\
H= & v_{1}\left(\Phi_{1}^{(1)} F-J_{1}^{(1)} H\right) \\
& +v_{2}\left(\Phi_{1}^{(2)} F-J_{2}^{(1)} H\right) \\
= & v_{1} \Phi_{2}^{(1)}+v_{2} \Phi_{2}^{(2)},
\end{aligned}
$$

the rest of rows can be obtained by analogy. As a result, we obtain $\Phi=v_{1} \Phi^{(1)}+v_{2} \Phi^{(2)}$; clearly, this relation can be generalized to an arbitrary vector of weight coefficients $v=\left(v_{1}, \ldots, v_{N}\right)$.

Assume that the value $k$ is minimal, satisfying the condition (20) and the set of solutions of (19) is represented in the form (21); note that the value $N=1$ is possible. To find the vector $v=\left(v_{1}, \ldots, v_{N}\right)$, taking into account the nonlinear term, rewrite the last equation in (5) in the form

$$
A=A_{* 1}\left(\begin{array}{c}
\Phi_{1} \\
\vdots \\
\Phi_{k}
\end{array}\right)+A_{* 2} H
$$

where $A_{*}=\left(\begin{array}{ll}A_{* 1} & A_{* 2}\end{array}\right)$. Set

$$
\Phi_{1}^{\Sigma}=\left(\begin{array}{c}
\Phi_{1}^{(1)} \\
\vdots \\
\Phi_{1}^{(N)}
\end{array}\right), \ldots, \quad \Phi_{k}^{\Sigma}=\left(\begin{array}{c}
\Phi_{k}^{(1)} \\
\vdots \\
\Phi_{k}^{(N)}
\end{array}\right)
$$

and rewrite (22) in the form

$$
A=A_{* 1}\left(\begin{array}{c}
v \Phi_{1}^{\Sigma} \\
\vdots \\
v \Phi_{k}^{\Sigma}
\end{array}\right)+A_{* 2} H
$$

By analogy with (6), Eqn. (23) has a solution if

$$
\operatorname{rank}\left(\begin{array}{c}
\Phi_{1}^{\Sigma} \\
\vdots \\
\Phi_{k}^{\Sigma} \\
H
\end{array}\right)=\operatorname{rank}\left(\begin{array}{c}
\Phi_{1}^{\Sigma} \\
\vdots \\
\Phi_{k}^{\Sigma} \\
H \\
A
\end{array}\right) .
$$

Assuming that (24) holds, at first consider the simple case when $A$ is a row matrix. Here, (23) can be represented in the form

$$
A=\left(\begin{array}{lll}
a_{1} v & \ldots & a_{k} v
\end{array}\right)\left(\begin{array}{c}
\Phi_{1}^{\Sigma} \\
\vdots \\
\Phi_{k}^{\Sigma}
\end{array}\right)+A_{* 2} H,
$$

where $A_{* 1}=\left(\begin{array}{lll}a_{1} & \ldots & a_{k}\end{array}\right)$, or in the form

$$
A=A_{v}\left(\begin{array}{c}
\Phi_{1}^{\Sigma} \\
\vdots \\
\Phi_{k}^{\Sigma}
\end{array}\right)+A_{* 2} H
$$

where $A_{v}$ is considered an unknown matrix. Note that if $N=1$, then $A_{* 1}=A_{v}$. Solve this algebraic equation and find the matrices $A_{v}$ and $A_{* 2}$. If $A_{v}$ can be represented in the form

$$
A_{v}=\left(\begin{array}{lll}
a_{1} v & \ldots & a_{k} v
\end{array}\right)
$$

for some coefficients $a_{1}, \ldots, a_{k}$ and the vector $v=$ $\left(v_{1}, \ldots, v_{N}\right)$, then stop, the results are the matrices $A_{* 1}=\left(a_{1} \ldots a_{k}\right)$ and $A_{* 2}$ and the vector $v$ of the weight coefficients. Then we find the matrix $R$ and the rows of matrices $J$ and $\Phi$ from the relations

$$
\begin{aligned}
R & =\sum_{i=1}^{N} v_{i} R^{(i)}, & J_{j} & =\sum_{i=1}^{N} v_{i} J_{j}^{(i)}, \\
\Phi_{j} & =\sum_{i=1}^{N} v_{i} \Phi_{j}^{(i)}, & j & =1,2, \ldots, k,
\end{aligned}
$$

and the matrices $G_{*}=\Phi G$ and $C_{*}=\Phi C$. As a result, the nonlinear observer described by the model (3) has been constructed.

If the matrix $A$ has several rows (25) is solved for all rows with some coefficients $a_{1}, \ldots, a_{k}$ specific for each row and the vector $v$ which is the same for all rows. If the condition (24) does not hold or the matrix $A_{v}$ cannot be represented in the required form, the dimension $k$ should be increased and the described procedure is repeated.

Let $\Phi$ be the matrix with rows found from (27), which guarantees disturbance decoupling and fault sensitivity for the linear part. Since the nonlinear part is constructed based on this matrix $\left(C_{*}=\Phi C\right.$ and the matrices $A_{* 1}$ and $A_{* 2}$ are found from (25)), then the nonlinear observer is disturbance decoupled and fault sensitive as well. Besides, if faults are not masked by disturbances in the linear systems, i.e., if the conditions $\Phi L=0$ and $\Phi D(x, u) \neq 0$ hold, then the faults are not masked by disturbances in the nonlinear systems.

Note that unlike the basic procedure of the LDA, where the nonlinear term is added to the existing linear part, the approach considered requires finding the matrices, describing the linear part, from the start. 


\section{Robustness}

5.1. Linear systems. If for all $k<n$ Eqn. (19) has no solutions, the disturbance decoupled linear observer cannot be designed and one has to use robust methods. From (19) it follows that the problem of minimizing the contribution of the disturbances in the residual reduces to minimizing the norm $\left\|\left(\begin{array}{lllll}R & -J_{1} & \ldots & -J_{k}\end{array}\right) B_{1}^{(k)}\right\|_{F}$ under the condition (9).

To solve this problem, we suggest to find the minimal value of the dimension $k$ when (9) has several linearly independent solutions in the form (21). It follows from Theorem 1 that an arbitrary linear combination of the rows of the matrix $W$ with the vector of weight coefficients $w=\left(w_{1}, \ldots, w_{N}\right)$ yields some solution as well. The problem is to find the vector $w$ such that $\|w\|=1$ and the norm $\left\|w W B_{1}^{(k)}\right\|_{F}$ is minimal. The constraint $\|w\|=1$ is used to avoid the trivial solution $w=0$.

To solve this problem, find the singular value decomposition of the matrix $W B_{1}^{(k)}$

$$
W B_{1}^{(k)}=U_{B} \Sigma_{B} V_{B}
$$

where $U_{B}$ and $V_{B}$ are orthogonal matrices,

$$
\Sigma_{B}=\left(\operatorname{diag}\left(\sigma_{1}, \ldots, \sigma_{c}\right) 0\right)
$$

or

$$
\Sigma_{B}=\left(\begin{array}{c}
\operatorname{diag}\left(\sigma_{1}, \ldots, \sigma_{c}\right) \\
0
\end{array}\right)
$$

depending on the numbers of rows and columns of the matrix $W B_{1}^{(k)}, c=\min (N, k), 0 \leq \sigma_{1} \leq \cdots \leq \sigma_{s}$ are the singular values of the matrix $W B_{1}^{(k)}$ ordered by magnitude (Low et al., 1996). Choose the first transposed column of the matrix $U_{B}$ as a vector of the weight coefficients $w=\left(w_{1}, \ldots, w_{N}\right)$. It follows from singular value decomposition and properties of orthogonal matrices that the norm of the matrix $w W B_{1}^{(k)}$ is equal to the minimal singular value $\sigma_{1}$.

Theorem 2. The vector $w=\left(w_{1}, \ldots, w_{N}\right)$ yields an optimal solution with the minimal norm of the vector

$$
\left(\begin{array}{llll}
R & -J_{1} & \ldots & -J_{k}
\end{array}\right) B_{1}^{(k)} .
$$

Proof. This result follows immediately from the choice of the vector $w=\left(w_{1}, \ldots, w_{N}\right)$ and properties of the singular value decomposition (Low et al., 1996).

Note that if $\sigma_{1}=0$, then $w W B_{1}^{(k)}=0$. This means that the linear combination of solutions represented by rows of the matrix $W$ with the vector of weight coefficients $w=\left(w_{1}, \ldots, w_{N}\right)$ yields the disturbance decoupled solution. If $\sigma_{1} \neq 0$, such a linear combination yields the solution with the minimal value of the norm $\left\|\left(\begin{array}{llll}R & -J_{1} & \ldots & -J_{k}\end{array}\right) B_{1}^{(k)}\right\|_{F}$.

Thus, the linear combination of solutions, represented by the rows of the matrix $W$ with the weight coefficients $w_{1}, \ldots, w_{s}$, yields the optimal solution with matrices $R, J$ and $\Phi$ found from (27) with $v=w$. Then the matrix $G_{*}=\Phi G$ is defined, and the robust linear observer has been built. Notice that this solution is optimal for a given dimension of the observer; if this dimension increases, the solution can be improved in the sense of the norm $\left\|\left(\begin{array}{llll}R & -J_{1} & \ldots & -J_{k}\end{array}\right) B_{1}^{(k)}\right\|_{F}$.

If $\Phi D(x(t), u(t))=0$ for all $(x(t), u(t)) \in X \times U$, then another transposed column of the matrix $U_{B}$ should be chosen as the vector of the weight coefficient $w=$ $\left(w_{1}, \ldots, w_{N}\right)$.

5.2. Nonlinear systems. The main idea to construct the nonlinear robust observer is as follows. We find all solutions satisfying the conditions (9), (24), and (26) and obtain the optimal solution according to Section 5.1.

The algorithm below constructs the robust nonlinear observer based on the singular value decomposition.

$\overline{\text { Algorithm 2. Robust nonlinear observer design. }}$
Step 1. Find the minimal value of $k$ for which (9) has
several linearly independent solutions.

Step 2. Find the set of all linearly independent solutions of (9) in the form (21).

Step 3. Find matrices $\Phi_{1}^{(1)}, \ldots, \Phi_{k}^{(1)}, \ldots, \Phi_{k}^{(N)}$ from (7) and check the condition (24). If it holds, solve (25) and check the condition (26). Let $b$ be the number of solutions satisfying the conditions (9), (24), and (26).

Step 4. If $b>1$, construct matrices $W$ and $B_{1}^{(k)}$, choose the vector $w=\left(w_{1}, w_{2}, \ldots, w_{N}\right)$, solve (25) and construct the nonlinear observer with minimal sensitivity to the disturbances. If $b=1$, the solution satisfying the conditions (9), (24), and (26) is unique for a given $k$. To improve the quality of this solution, one may take $k:=k+1$ and go to Step 2. If $b=0$, set $k:=k+1$ and go to Step 2 .

A shortcoming of the suggested approach is that the minimal sensitivity to the disturbances may be followed by low sensitivity to the faults. To overcome this difficulty, we suggest to consider the norm $\|\Phi D\|_{F}$, estimating the contribution of the faults in the residual, on the analogy of the norm $\|\Phi L\|_{F}$, estimating the contribution of the disturbances to the residual, and minimize the performance index

$$
J=\frac{\|\Phi L\|_{F}^{2}}{\|\Phi D\|_{F}^{2}}
$$

The problem can be solved by the method considered by Alcorta-Garcia and Frank (1997) for parity relations; it may be reduced to the generalized eigenvector and eigenvalue problem of the matrices $W B_{1}^{(k)}\left(W B_{1}^{(k)}\right)^{T}$ 
and $W B_{2}^{(k)}\left(W B_{2}^{(k)}\right)^{T}$, where

$$
B_{2}^{(k)}=\left(\begin{array}{ccccc}
H D & H F D & H F^{2} D & \ldots & H F^{k-1} D \\
0 & H D & H F D & \ldots & H F^{k-2} D \\
0 & 0 & H D & \ldots & H F^{k-3} D \\
\ldots \ldots & \ldots \ldots \ldots \ldots \ldots \ldots & \ldots \ldots \ldots \\
0 & 0 & 0 & \ldots & 0
\end{array}\right)
$$

The best choice for the row $\left(\begin{array}{lllll}R & -J_{1} & \ldots & -J_{k}\end{array}\right)$ in this case is the transposed generalized eigenvector, corresponding to the minimal generalized eigenvalue. Recall that the generalized eigenvector and eigenvalue problem for the matrices $Q_{1}$ and $Q_{2}$ is formulated as follows: find a vector $w$ and a number $\lambda$ such that $Q_{1} w=$ $\lambda Q_{2} w$.

To summarize, the algorithm suggested below constructs the disturbance decoupled observer or the robust observer based on the generalized eigenvectors and eigenvalues.

Algorithm 3. Nonlinear observer design.

Step 1. Find the minimal dimension $k$ from (10).

Step 2. Construct the matrix $B_{1}^{(k)}$.

Step 3. If the condition (20) holds, from (19) find the row $\left(R-J_{1} \ldots-J_{k}\right)$ and construct the disturbance decoupled linear observer. Otherwise, go to Step 5.

Step 4. To construct the nonlinear observer, find a set of all linearly independent solutions of (19) in the form (21), find matrices $\Phi_{1}^{(1)}, \ldots, \Phi_{k}^{(1)}, \ldots, \Phi_{k}^{(N)}$ from (7) and check the condition (24). If it holds, solve (25) and check the condition (26). If it holds, find the matrices, describing the nonlinear observer and stop. Otherwise, go to Step 5.

Step 5. Set $k:=k+1$ and repeat Steps $2-4$. If the conditions (20), (24), and (26) hold, construct the disturbance decoupled nonlinear observer; otherwise, repeat Step 5. When $k=n$, go to Step 6 to find the robust solution.

Step 6. Find the minimal value $k$ for which (9) has several linearly independent solutions, construct matrices $W$, $B_{1}^{(k)}$, and $B_{2}^{(k)}$, choose the vector $w=\left(w_{1}, w_{2}, \ldots, w_{N}\right)$ and construct the linear observer with the minimal value of the performance index $J$.

Step 7. Use Algorithm 2 after replacing the matrices $W$ and $B_{1}^{(k)}$ in Step 4 by $W, B_{1}^{(k)}$, and $B_{2}^{(k)}$, respectively.

\section{Illustrative example}

Consider the control system:

$$
\begin{aligned}
x_{1}^{+} & =x_{1}+x_{2}-x_{3} u_{1}+L_{1} \rho, \\
x_{2}^{+} & =0.5 x_{1}-x_{3}+\gamma \operatorname{abs}\left(1.7831 x_{4}-x_{2}\right)+L_{2} \rho, \\
x_{3}^{+} & =x_{1}+x_{2}-x_{4}+u_{2}+L_{3} \rho, \\
x_{4}^{+} & =-0.1 x_{1}+x_{4}+L_{4} \rho, \\
y_{1} & =x_{1}, \quad y_{2}=x_{3} .
\end{aligned}
$$

The nominal value of the parameter $\gamma$ is equal to 1 . The system has the logic-dynamic description with the following matrices and nonlinearities:

$$
\begin{aligned}
& F=\left(\begin{array}{cccc}
1 & 1 & 0 & 0 \\
0.5 & 0 & -1 & 0 \\
1 & 1 & 0 & -1 \\
-0.1 & 0 & 0 & 1
\end{array}\right) \\
& H=\left(\begin{array}{llll}
1 & 0 & 0 & 0 \\
0 & 0 & 1 & 0
\end{array}\right) \text {, } \\
& D(x, u)=\left(\begin{array}{c}
0 \\
\operatorname{abs}\left(1.7831 x_{4}-x_{2}\right) \\
0 \\
0
\end{array}\right) \text {, } \\
& G=\left(\begin{array}{ll}
0 & 0 \\
0 & 0 \\
0 & 1 \\
0 & 0
\end{array}\right), \quad C=\left(\begin{array}{cc}
-1 & 0 \\
0 & 1 \\
0 & 0 \\
0 & 0
\end{array}\right), \\
& \varphi_{2}\left(A_{2} x, u\right)=\operatorname{abs}\left(1.7831 x_{4}-x_{2}\right) \text {, } \\
& A_{1}=\left(\begin{array}{llll}
0 & 0 & 1 & 0
\end{array}\right), \\
& A_{2}=\left(\begin{array}{llll}
0 & -1 & 0 & 1.7831
\end{array}\right) \text {. }
\end{aligned}
$$$$
\varphi_{1}\left(A_{1} x, u\right)=x_{3} u_{1} \text {, }
$$

Consider at first the case

$$
\begin{aligned}
L^{(1)} & =\left(\begin{array}{lllll}
L_{1} & L_{2} & L_{3} & L_{4}
\end{array}\right)^{T} \\
& =\left(\begin{array}{llll}
0.2060 & 0.7960 & 0.0905 & 0.5619
\end{array}\right)^{T}
\end{aligned}
$$

and construct the disturbance $\rho$ decoupled linear observer. It can be shown that the condition (20) holds for $k=2$ since $\operatorname{rank}\left(V^{(2)} B_{1}^{(2)}, 0.0001\right)=5$ and $l(k+1)=6$; the matrices $V^{(2)}$ and $B_{1}^{(2)}$ are as follows:

$$
V^{(2)}=\left(\begin{array}{cccc}
1.5 & 1 & -1 & 0 \\
1.6 & 1 & -1 & -1 \\
1 & 1 & 0 & 0 \\
1 & 1 & 1 & -1 \\
1 & 0 & 0 & 0 \\
0 & 0 & 1 & 0
\end{array}\right) \text {, }
$$




$$
B_{1}^{(2)}=\left(\begin{array}{cc}
0.2060 & 1.0020 \\
0.0905 & 0.4401 \\
0 & 0.2060 \\
0 & 0.0905 \\
0 & 0 \\
0 & 0
\end{array}\right) \text {. }
$$

One can check that the row

$$
\begin{aligned}
& \left(\begin{array}{lll}
R & -J_{1} & -J_{2}
\end{array}\right) \\
& =\left(\begin{array}{lll}
0.2604 & -0.5929 & -0.2604
\end{array}\right. \\
& \begin{array}{lll}
0.5929 & 0.2255 & -0.3325)
\end{array}
\end{aligned}
$$

is the solution of (19) and

$$
\begin{aligned}
R & =\left(\begin{array}{ll}
0.2604 & -0.5929
\end{array}\right), \\
J & =\left(\begin{array}{cc}
0.2604 & -0.5929 \\
-0.2255 & 0.3325
\end{array}\right) .
\end{aligned}
$$

From (4) and (7) it follows that

$$
\Phi=\left(\begin{array}{cccc}
0.2604 & 0 & -0.5929 & 0 \\
-0.5929 & -0.3325 & 0.5929 & 0.5929
\end{array}\right)
$$

and

$$
G_{*}=\left(\begin{array}{cc}
0 & -0.5929 \\
0 & 0.5929
\end{array}\right) .
$$

Since the row $A_{1}$ can be expressed via $H$, consider the second nonlinearity $\varphi_{2}\left(A_{2} x, u\right)=\operatorname{abs}\left(1.7831 x_{4}-\right.$ $\left.x_{2}\right)$. Since $\operatorname{rank}\left(V^{(2)} B_{1}^{(2)}\right)=5$ and $l(k+1)=6$, (19) has a unique solution and $N=1$. As a result, the relation (25) for $\varphi_{2}\left(A_{2} x, u\right)$ takes the form

$$
\begin{aligned}
& \left(\begin{array}{llll}
0 & -1 & 0 & 1.7831
\end{array}\right) \\
& =A_{v}\left(\begin{array}{cccc}
0.2604 & 0 & -0.5929 & 0 \\
-0.5929 & -0.3325 & 0.5929 & 0.5929
\end{array}\right) \\
& +A_{* 2}\left(\begin{array}{cccc}
1 & 0 & 0 & 0 \\
0 & 0 & 1 & 0
\end{array}\right) \text {, }
\end{aligned}
$$

which gives

$$
\begin{gathered}
A_{v}=\left(\begin{array}{ll}
0 & 3.0075
\end{array}\right), \\
A_{* 2}=\left(\begin{array}{ll}
1.7831 & -1.7831
\end{array}\right) .
\end{gathered}
$$

Since $N=1$, we have $A_{* 1}=A_{v}$.

Since

$$
\Phi D(x, u)=-0.3325 \operatorname{abs}\left(1.7831 x_{4}-x_{2}\right) \neq 0
$$

for all $(x, u) \in X \times U$ except on the set

$$
S=\left\{(x, u) \mid 1.7831 x_{4}-x_{2}=0, u \in U\right\},
$$

the observer is sensitive to the fault. Calculate

$$
C_{*}=\Phi C=\left(\begin{array}{cc}
-0.2604 & 0 \\
0.5929 & -0.3325
\end{array}\right)
$$

and set

$$
\begin{aligned}
x_{* 1}:= & 0.2604 x_{1}-0.5929 x_{3}, \\
x_{* 2}:= & -0.5929 x_{1}-0.3325 x_{2} \\
& +0.5929 x_{3}+0.5929 x_{4} .
\end{aligned}
$$

As a result, we obtain the description of the disturbance decoupled nonlinear observer:

$$
\begin{aligned}
x_{* 1}^{+}= & x_{* 2}+0.2604 y_{1}-0.5929 y_{2}-0.2604 y_{2} u_{1} \\
& -0.5929 u_{2}, \\
x_{* 2}^{+}= & -0.2255 y_{1}+0.3325 y_{2}+0.5929 y_{2} u_{1} \\
& +0.5929 u_{2}-0.3325 \mathrm{abs}\left(3.0075 x_{* 2}\right. \\
& \left.+1.7831 y_{1}-1.7831 y_{2}\right), \\
r= & 0.2604 y_{1}-0.5929 y_{2}-x_{* 1} .
\end{aligned}
$$

Next, to illustrate the process of obtaining the robust solution, assume that the disturbances are described by the matrix $L^{(2)}=\left(\begin{array}{llll}0.2 & 0.5 & 0.2 & 0.5\end{array}\right)^{T}$. It can be shown that (19) has no solutions for all $k<4$, so we need to use the robust methods. It can be also shown that (9) has two solutions for $k=2$ :

$$
\begin{aligned}
& \left(\begin{array}{lll}
R^{(1)} & -J_{1}^{(1)} & -J_{2}^{(1)}
\end{array}\right) \\
& =\left(\begin{array}{ll}
0.2604 & -0.5929
\end{array}\right. \\
& \begin{array}{llll}
-0.2604 & 0.5929 & 0.2255 & -0.3325),
\end{array} \\
& \left(\begin{array}{lll}
R^{(2)} & -J_{1}^{(2)} & -J_{2}^{(2)}
\end{array}\right) \\
& =\left(\begin{array}{ll}
0.5443 & 0.0248
\end{array}\right. \\
& \begin{array}{llll}
-0.5443 & -0.0248 & -0.2870 & 0.5691) .
\end{array}
\end{aligned}
$$

Construct the matrices

$$
\begin{aligned}
& W=\left(\begin{array}{ccc}
0.2604 & -0.5929 & -0.2604 \\
0.5443 & 0.0248 & -0.5443
\end{array}\right. \\
& \left.\begin{array}{ccc}
0.2255 & -0.3325 & 0.5929 \\
-0.2870 & 0.5691 & -0.0248
\end{array}\right), \\
& B_{1}^{(2)}=\left(\begin{array}{cccccc}
0.2 & 0.2 & 0 & 0 & 0 & 0 \\
0.7 & 0.2 & 0.2 & 0.2 & 0 & 0
\end{array}\right)^{T} .
\end{aligned}
$$

Find the matrix $U_{B}$ from the singular value decomposition of $W B_{1}^{(2)}$ :

$$
U_{B}=\left(\begin{array}{cc}
0.3447 & 0.9387 \\
0.9387 & -0.3447
\end{array}\right)
$$

The last column of this matrix corresponds to the minimal singular value $\sigma=0.1056$; therefore,

$$
w=\left(\begin{array}{ll}
0.9387 & -0.3447
\end{array}\right) .
$$

Compute

$$
\begin{aligned}
& R=\left(\begin{array}{ll}
0.0568-0.5651
\end{array}\right), \\
& J=\left(\begin{array}{cc}
0.0568 & -0.5651 \\
-0.3106 & 0.5083
\end{array}\right),
\end{aligned}
$$




$$
\begin{aligned}
\Phi & =\left(\begin{array}{cccc}
0.0568 & 0 & -0.5651 & 0 \\
-0.5651 & -0.5083 & 0.5651 & 0.5651
\end{array}\right), \\
G_{*} & =\left(\begin{array}{cc}
0 & -0.5651 \\
0 & 0.5651
\end{array}\right), \\
\Phi L^{(2)} & =\left(\begin{array}{c}
-0.1017 \\
0.0284
\end{array}\right) .
\end{aligned}
$$

Note that $\left\|\Phi L^{(2)}\right\|=\sigma=0.1056$. The description of the linear observer with minimal sensitivity to the disturbances is as follows:

$$
\begin{aligned}
x_{* 1}^{+} & =x_{* 2}+0.0568 y_{1}-0.5651 y_{2}-0.5651 u_{2}, \\
x_{* 2}^{+} & =-0.3106 y_{1}+0.5083 y_{2}+0.5651 u_{2}, \\
r & =0.0568 y_{1}-0.5651 y_{2}-x_{* 1} .
\end{aligned}
$$

Based on Algorithm 1, one can show that $b=1$ so the solution satisfying the conditions (9), (24), and (26) is unique for $k=2$ with

$$
\begin{aligned}
R & =\left(\begin{array}{ll}
0.2604 & -0.5929
\end{array}\right), \\
J & =\left(\begin{array}{cc}
0.2604 & -0.5929 \\
-0.2255 & 0.3325
\end{array}\right) .
\end{aligned}
$$

The description of the nonlinear observer almost coincides with (28); since

$$
\Phi L^{(2)}=\left(\begin{array}{c}
-0.0665 \\
0.1302
\end{array}\right),
$$

we get $\left\|\Phi L^{(2)}\right\|=0.1462>\sigma$.

To improve this solution, one may take $k=3$ that yields a better solution with

$$
\begin{aligned}
R & =\left(\begin{array}{ll}
-0.1115 & 0.4518
\end{array}\right), \\
J & =\left(\begin{array}{cc}
-0.1675 & 0.6281 \\
0.2713 & -0.5165 \\
-0.0778 & 0.1202
\end{array}\right) .
\end{aligned}
$$

It can be shown that

$$
\begin{aligned}
& \Phi=\left(\begin{array}{cccc}
-0.1115 & 0 & 0.4518 & 0 \\
0.5078 & 0.3403 & -0.6281 & -0.4518 \\
-0.1763 & -0.1203 & 0.1762 & 0.1763
\end{array}\right) \\
& C_{*}=\left(\begin{array}{cc}
0.1115 & 0 \\
-0.5078 & 0.3403 \\
0.1763 & -0.1203
\end{array}\right) \text {, } \\
& G_{*}=\left(\begin{array}{cc}
0 & 0.4518 \\
0 & -0.6281 \\
0 & 0.1762
\end{array}\right) \text {, }
\end{aligned}
$$

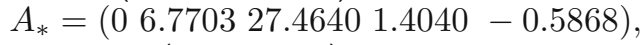

$$
\begin{aligned}
& \Phi L^{(2)}=\left(\begin{array}{c}
0.0681 \\
-0.0798 \\
0.0280
\end{array}\right)
\end{aligned}
$$

and then $\left\|\Phi L^{(2)}\right\|=0.1086$.
The description of the there-dimensional nonlinear observer is as follows:

$$
\begin{aligned}
x_{* 1}^{+}= & x_{* 2}-0.1675 y_{1}+0.6281 y_{2}+0.4518 u_{2} \\
& +0.1115 y_{2} u_{1}, \\
x_{* 2}^{+}= & x_{* 3}+0.2713 y_{1}-0.5165 y_{2}-0.6281 u_{2} \\
& -0.5078 y_{2} u_{1}+0.3403 \operatorname{abs}\left(6.7703 x_{* 2}\right. \\
& \left.+27.4640 x_{* 3}+1.4040 y_{1}-0.5868 y_{2}\right), \\
x_{* 3}^{+}= & -0.0778 y_{1}+0.1202 y_{2}+0.1762 u_{2} \\
& +0.1763 y_{2} u_{1}-0.1203 \operatorname{abs}\left(6.7703 x_{* 2}\right. \\
& \left.+27.4640 x_{* 3}+1.4040 y_{1}-0.5868 y_{2}\right), \\
r= & -0.1115 y_{1}+0.4518 y_{2}-x_{* 1} .
\end{aligned}
$$

For simulation, consider the observers (28) with $K=\left(\begin{array}{ll}0 & 0.1\end{array}\right)^{T}$ and (29) with $K=\left(\begin{array}{lll}0 & 0 & 0\end{array}\right)^{T}$. The initial conditions of the system are zero, the initial conditions of the observer $(28)$ are $x_{* 1}(0)=x_{* 2}(0)=$ 0.01 , and those of the observer (29) are zero. The control are $u_{1}(t)=0.1 \sin (i / 10), u_{2}(t)=0.2$ if $t<30, u_{2}(t)=$ 0.1 if $t \geq 30$. The disturbance $\rho(t)$ has uniform random distribution on $(-0.5,0.5)$. The fault is modeled by abruptly changing the parameter $\gamma$ at $t=120$ from $\gamma=1$ to $\gamma=0.7$ onward.

Simulation results are shown in Figs. 1-3. Figure 1 shows that the observer is disturbance decoupled, Figures 2 and 3 show the behavior of robust observers. The observer (29) reveals less sensitivity to the disturbances than (28).

\section{Conclusions}

In the present paper, the problem of fault detection and isolation in technical systems described by dynamic

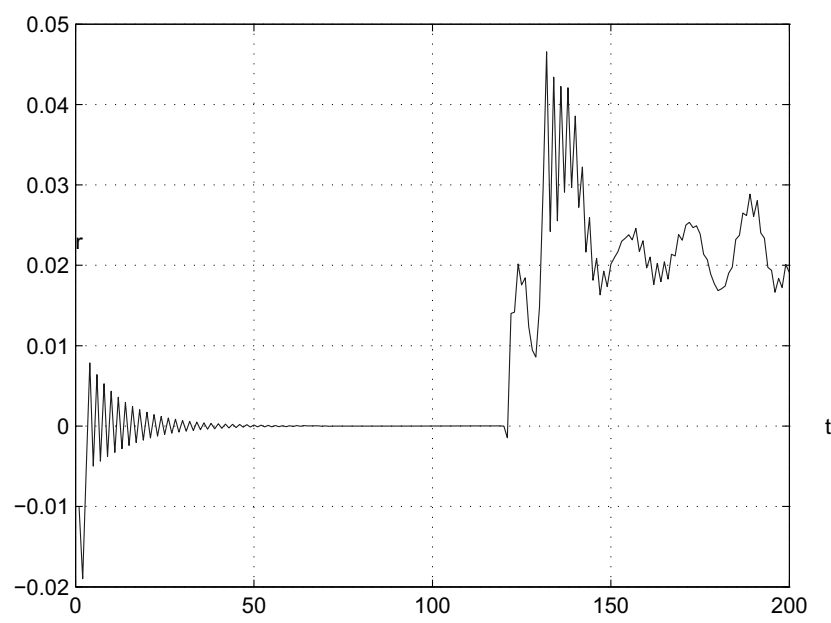

Fig. 1. Residual behavior for the observer (28) and $L^{(1)}=$ $\left(\begin{array}{llll}0.2060 & 0.7960 & 0.0905 & 0.5619\end{array}\right)^{T}$. 


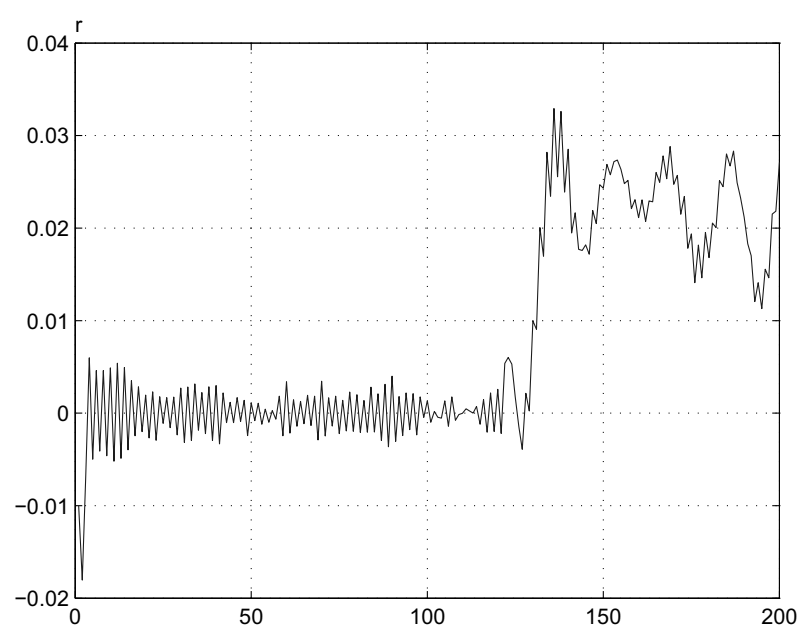

Fig. 2. Residual behavior for the observer (28) and $L^{(2)}=$ $\left(\begin{array}{llll}0.2 & 0.5 & 0.2 & 0.5\end{array}\right)^{T}$.

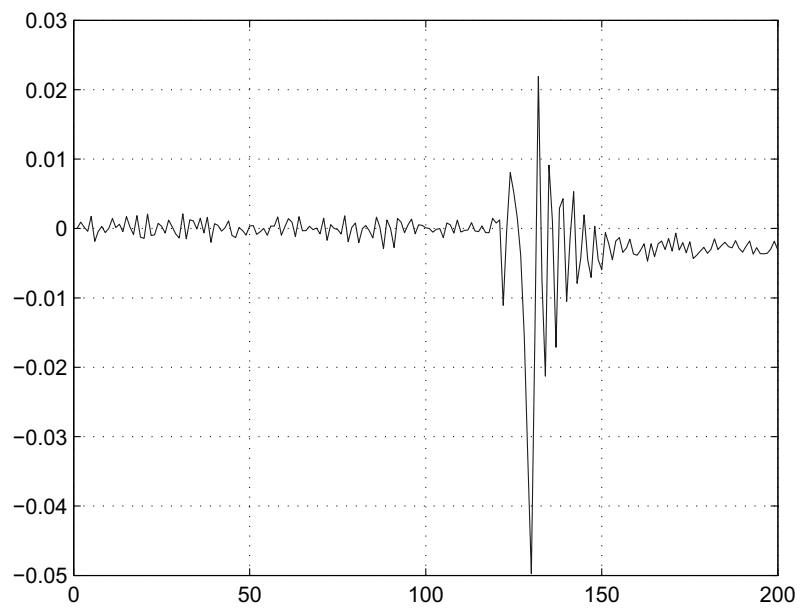

Fig. 3. Residual behavior for the observer (29) and $L^{(2)}=$ $\left(\begin{array}{llll}0.2 & 0.5 & 0.2 & 0.5\end{array}\right)^{T}$.

models with non-differentiable nonlinearities has been considered. Some method to construct disturbance decoupled observers or having minimal sensitivity to disturbances have been suggested. The method is based on the so-called logic-dynamic approach which allows us to solve the problem under consideration by linear methods. This approach can be applied for both continuous-time and discrete-time systems.

\section{Acknowledgment}

This work was supported by the Russian Scientific Foundation, project no. 16-19-00046.

\section{References}

Alcorta-Garcia, E. and Frank, P. (1997). Deterministic nonlinear observerbased approach to fault diagnosis: A survey, Control Engineering Practice 5(5): 663-670.

Boulkroune, B., Djemili, I., Aitouche, A., and Cocquempot, V. (2013). Robust nonlinear observer design for actuator fault detection in diesel engines, International Journal of Applied Mathematics and Computer Science 23(3): 557-569, DOI: 10.2478/amcs-2013-0042.

Blanke, M., Kinnaert, M., Lunze, J., and Staroswiecki, M. (2006). Diagnosis and Fault-Tolerant Control, Springer, Berlin.

Caccavale, F. and Villiani, L. (Eds.) (2002). Fault Diagnosis and Tolerance for Mechatronic Systems: Recent Advances, Springer, Berlin.

Ding, S. (2013). Model-Based Fault Diagnosis TechniquesDesign Schemes, Algorithms and Tools, 2nd Edn., Springer, London.

Ducard, G.J.J. (2015). SMAC-FDI: A single model active fault detection and isolation system for unmanned aircraft, International Journal of Applied Mathematics and Computer Science 25(1): 189-201, DOI: 10.1515/amcs-2015-0014.

Filaretov V., Vukobratovic M., and Zhirabok A. (2003). Parity relation approach to fault diagnosis in manipulation robots, Mechatronics 13(2): 141-152.

Filaretov, V. and Zhirabok, A. (2006). Logic-dynamic approach to fault diagnosis in mechatronic systems, International Journal of Advanced Robotic Systems 3(4): 285-294.

Frank, P. (1990). Fault diagnosis in dynamic systems using analytical and knowledge-based redundancy-A survey and some new results, Automatica 26(3): 459-474.

Gertler, J. (1993). Residual generation in model-based fault diagnosis, Theory and Advanced Technology 9(1): 259-285.

Low, X. and Willsky, A. and Verghese, G. (1996). Optimally robust redundancy relations for failure detection in uncertain systems, Automatica 22(3): 333-344.

Patton, R. (1994). Robust model-based fault diagnosis: The state of the art, IFAC Symposium SAFEPROCESS'2004, Espoo, Finland, pp. 1-24.

Patton, R., Frank, P. and Clark, R. (2000). Issues of Fault Diagnosis for Dynamic Systems, Springer, London.

Persis, D.C. and Isidori, A. (2001). A geometric approach to nonlinear fault detection and isolation, IEEE Transactions on Automatic Control AC-46(6): 853-865.

Russell, E., Chiang, L., and Chiang, L. (2001). Fault Detection and Diagnosis in Industrial Systems, Springer, Berlin.

Samy, I., Postlethwaite, I. and Gu, D. (2011). Survey and application of sensor fault detection and isolation schemes, Control Engineering Practice 19(5): 658-674.

Schreier, G., Ragot, J., Patton, R., and Frank, F. (1997). Observer design for a class of nonlinear systems, IFAC Symposium SAFEPROCESS'1997, Hull, UK, pp. 498-503. 
Simani, S., Fantuzzi, C., and Patton, R. (2002). Model-Based Fault Diagnosis in Dynamic Systems Using Identification, Springer, Berlin.

Shumsky, A. and Zhirabok, A. (2006). Nonlinear diagnostic filter design: Algebraic and geometric points of view, International Journal of Applied Mathematics and Computer Science 16(1): 115-127.

Zhirabok, A. and Usoltsev, S. (2002). Fault diagnosis for nonlinear dynamic systems via linear methods, 15 th World Congress IFAC, Barcelona, Spain, (on CD ROM).

Zhirabok, A., Kucher, D., and Filaretov, V. (2010). Achieving robustness at diagnosis of nonlinear systems, Automation and Remote Control 71(1): 142-155.

Zhirabok, A. and Shumsky, A. (2010). Linear methods in observability and controllability of nonlinear systems, 8 th IFAC Symposium on Nonlinear Systems, Bologna, Italy, pp. 308-313.

Zhirabok, A. and Shumsky, A. (2013). Logic-dynamic approach to the robust fault diagnosis in nonlinear systems, 2013 Conference on Control and Fault Tolerant Systems, Nice, France, pp. 190-195.

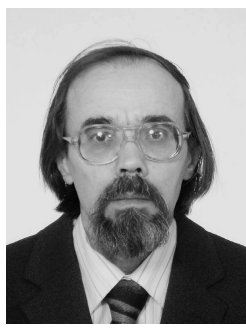

Alexey Zhirabok is a professor at Far Eastern Federal University and a chief researcher in the Institute of Marine Technology Problems, Russian Academy of Sciences (both in Vladivostok). He received his Candidate of Science $(\mathrm{PhD})$ degree in radiolocation and radionavigation from Leningrad (St. Petersburg) Electrotechnical Institute in 1978 and his DSc degree in automatic control from the Institute of Automation and Control Processes, Russian Academy of Sciences, in 1996. His research interests include nonlinear control theory with application to fault diagnosis and fault tolerant control.

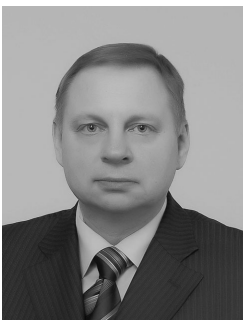

Alexey Shumsky is a professor at Far Eastern Federal University and a chief researcher in the Institute of Applied Mathematics, Russian Academy of Sciences (both in Vladivostok). $\mathrm{He}$ received his Candidate of Science $(\mathrm{PhD})$ degree in radiolocation and radionavigation from Leningrad (St. Petersburg) Electrotechnical Institute in 1985 and his DSc degree in automatic control from the Institute of Control Problems, Russian Academy of Sciences (Moscow), in 1996. His research interests include nonlinear control theory with application to fault diagnosis and fault tolerant control.

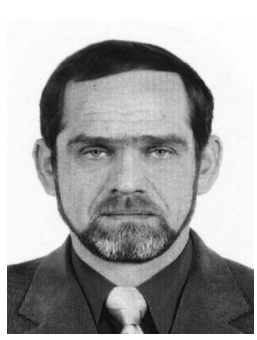

Sergey Solyanik is an associate professor at Far Eastern Federal University (Vladivostok). He received his Candidate of Science $(\mathrm{PhD})$ degree in radio and electronics from the same University in 2007. His research interests include fault diagnosis and fault tolerant control.

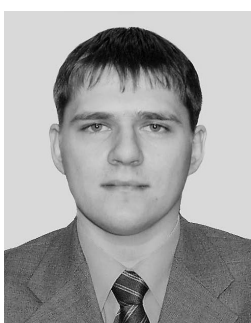

Alexey Suvorov is a postgraduate student at Far Eastern Federal University (Vladivostok). His research interests include fault diagnosis and fault tolerant control.

Received: 8 July 2016

Revised: 28 November 2016

Re-revised: 18 February 2017

Accepted: 10 March 2017 